\title{
Über Aldehydderivate der Rhodanine und ihre Spaltungsprodukte
}

\author{
I. Mitteilung \\ Von \\ Rudolf Andreasch \\ (Vorgelegt in der Sitzung am 18. April 1918)
}

Oxydation von Benzalphenylrhodanin.

Es sollte zunächst versucht werden, wie sich die Aldehydkondensationsprodukte der Rhodanine dem Brom gegenüber verhalten. Da dieselben eine Doppelbindung besitzen, so war eine Anlagerung von Brom an diese nicht unmöglich.

Einige Gramm von Benzalphenylrhodanin wurden in Eisessig in der Siedehitze gelöst und zur Lösung mit Eisessig verdünntes Brom zutropfen gelassen. Beim Sieden verschwand das Brom ziemlich rasch; es zeigte sich aber sofort, daß die verbrauchte Brommenge ein Molekül weit überschritt. Eine Gasentwicklung war nicht zu bemerken. Sobald das Brom auch bei längerem Sieden nicht mehr verschwand, wurde abgekühlt, wobei sich ein Brei sehr feiner Nadeln abschied; aus den Mutterlaugen ließ sich durch Abdestillieren des Eisessigs noch mehr der Verbindung gewinnen. Der Körper wurde aus kochendem Alkohol und aus Aceton umkrystallisiert und bildete nach dem Absaugen eine weiße papierähnliche Masse vom Schmelzpunkt $209^{\circ}$. Der Körper war bromfrei und gab nach dem Kochen mit Lauge die violette Thioglykolsäurereaktion. Danach war es wahrscheinlich, daß nur eine Oxydation durch das Brom stattgefunden habe und 
daß der Körper nichts anderes als das bereits dargestellte Benzalphenylsenfölglykolid sei, ${ }^{1}$ was auch die Analyse bestätigte.

Analyse:

$0.123 g$ Substanz: $0.3075 g \mathrm{CO}_{2}, 0.0418 g \mathrm{H}_{2} \mathrm{O}$.

$0 \cdot 120 \mathrm{~g}$ Substanz: $0 \cdot 1006 \mathrm{~g} \mathrm{Ba} \mathrm{SO}_{4}$.

$0 \cdot 1944 \mathrm{~g}$ Substanz: $\left(18^{\circ}, 735 \mathrm{~mm}\right) 9 \mathrm{~cm}^{3} \mathrm{~N}$.

$\mathrm{C}_{16} \mathrm{H}_{11} \mathrm{O}_{2} \mathrm{NS}$ Ber. C $68 \cdot 30$, H 3.94, N 4.98, S $11 \cdot 40$.

Gef. C $68 \cdot 17, \mathrm{H} 3 \cdot 80, \mathrm{~N} 5 \cdot 07$, S $11 \cdot 50$.

Es wirkt also das Brom lediglich als Oxydationsmittel, indem es den Schwefel des Thiocarbonyls zu Schwefelsäure, welche in den Mutterlaugen leicht nachzuweisen ist, oxydiert und ihn durch Sauerstoff ersetzt:

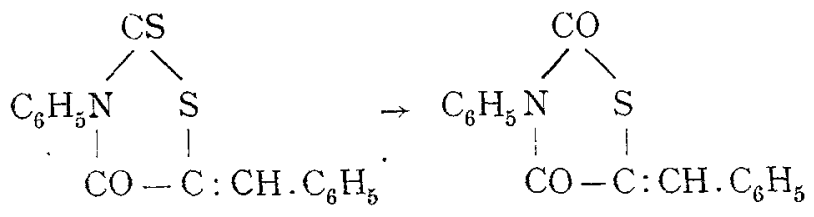

Ein gleicher Versuch mit Brom in Eisessiglösung wurde mit dem $o$-Nitrobenzalphenylhhodanin ausgeführt und wurden dabei gelbliche Krystalle erhalten, die nach dem Umkrystallisieren aus Alkohol und Aceton nahezu weiß wurden und einen Schmelzpunkt von $204^{\circ}$ aufwiesen. Da das betreffende $o$-Nitrobenzalphenylsenfölglykolid noch nicht bekannt war, stellte ich es durch Erhitzen von Phenylsenfölglykolid und o-Nitrobenzaldehyd in Eisessig her. Die Verbindung stellte farblose, lockere, wollige Nadeln dar, die denselben Schmelzpunkt wie die durch die Bromeinwirkung erhaltenen zeigten und bei der Analyse die verlangten Werte ergaben.

Analyse:

$0.1317 g$ Substanz; $0.284 \mathrm{~g} \mathrm{CO}_{2}, 0.0387 \mathrm{~g} \mathrm{H} \mathrm{H}_{2} \mathrm{O}$.

$\mathrm{C}_{16} \mathrm{H}_{10} \mathrm{O}_{4} \mathrm{~N}_{2} \mathrm{~S}$ Ber. C $58 \cdot 88, \mathrm{H} 3 \cdot 09$.

Gef. C $58 \cdot 81, \mathrm{H} 3 \cdot 29$.

1 Monatshefte für Chemie, 38, 125. In dieser Abhandlung ist der Schmelzpunkt der Verbindung durch einen Druckfehler zu $239^{\circ}$ statt $209^{\circ}$ angegeben. 
Versuche, in ähnlicher Weise von dem Rhodanin durch Oxydation mittels Brom zur Senfölessigsäure zu gelangen oder aus dem Äthylidenrhodanin von Nencki eine Äthylidensenfölessigsäure $\mathrm{zu}$ erhalten, führten $\mathrm{zu}$ keinen faßbaren Produkten.

Nach Abschluß der vorstehenden Versuche fiel mir eine Angabe in die Hände, nach welcher es Kallenberg' gelang, die aktive und inaktive Diäthyldithiocarbaminmalaminsäure durch Oxydation mit Permanganat in kohlensaurer Lösung in ähnlicher Weise zu entschwefeln. Es scheinen also saure Oxydationsmittel auf die Aldehydkondensationsprodukte der Rhodanine und verwandte Derivate in dem Sinne einzuwirken, daß der Thiocarbonylschwefel durch Sauerstoff ersetzt wird. Ich möchte auch darauf verweisen, daß es bereits im Jahre 1886 J. Ginsburg und St. Bondzyński² gelang, die Benzylidenrhodaninsäure oder, wie wir heute den Körper nennen, das Benzalrhodanin durch Erhitzen mit konzentrierter Schwefelsäure in ein entsprechendes Senfölessigsäurederivat umzuwandeln.

Unterwirft man das Phenylrhodanin selbst der Einwirkung von Brom in Eisessiglösung bei der Siedetemperatur des letzteren, so erhält man, allerdings nur in kleiner Menge, einen in feinen Blättchen krystallisierenden, bromhaltigen Körper, der abgesaugt das Ansehen von Mussivgold hat und einen Schmelzpunkt von $143^{\circ}$ besitzt. Der Körper wird noch weiter untersucht werden.

\section{$\beta$ - ̈̈thylrhodanin.}

Alle Aldehydkondensationsprodukte der Rhodanine enthalten eine Doppelbindung und es ließ sich daher annehmen, daß sie durch geeignete Reduktionsmittel an diese Wasserstoff anlagern würden. So sollte das Äthylidenrhodanin (I) in das ß-Äthylrhodanin (II) übergehen:

1 Ber. d. d. chem. Ges., 50, 99 und 100.

2 Ibid., 19, 113. 
<smiles>CC1SSNC1=O</smiles>

I.

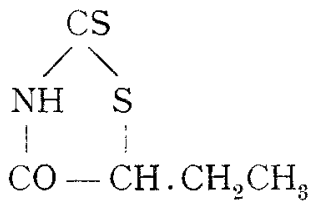

II.

Um die Eigenschaften dieser zu erwartenden Reduktionsprodukte kennen zu lernen, wurde zunächst das $\beta$-Äthylrhodanin durch Einwirkung von $\alpha$-Brombuttersäure auf Ammoniumdithiocarbamat dargestellt:<smiles>CCC(Br)C(Br)=S(NC(=O)Br)C(CC)CC</smiles>

Das feingeriebene Dithiocarbamat wurde in Alkohol suspendiert und mit der äquivalenten Menge $\alpha$-Brombuttersäureester zusammengebracht, wobei die eintretende Erwärmung die Reaktion anzeigte. Es wird nun einige Zeit am Rückflußkühler gekocht, der Alkohol zum Teil abdestilliert, die Flüssigkeit mit Wasser versetzt und das ausgeschiedene Öl in Äther aufgenommen. Der beim Abdestillieren des Äthers verbleibende Rückstand erstarrt bald, er wird zur Entfernung beigemengter schmieriger Bestandteile auf Ton aufgestrichen und die zurückbleibenden Krystalle in heißem Alkohol gelöst. Bei vorsichtigem Zusatz von Wasser entsteht anfangs eine Trübung, die sich bald in ein gelblichweißes Krystallpulver verwandelt, das mit folgendem Resultat analysiert wurde.

Analyse:

$0.1338 g$ Substanz: $0.1833 g \mathrm{CO}_{2}, 0.054 g \mathrm{H}_{2} \mathrm{O}$.

$0.173 \mathrm{~g}$ Substanz $\left(24^{\circ}, 732 \mathrm{~mm}\right) 14 \cdot 1 \mathrm{~cm}^{3} \mathrm{~N}$.

$\mathrm{C}_{5} \mathrm{H}_{7} \mathrm{ONS}_{2}$ Ber. C $37 \cdot 23, \mathrm{H} 4 \cdot 38, \mathrm{~N} 8 \cdot 69$. Gef. C $37 \cdot 36, \mathrm{H} 4 \cdot 52, \mathrm{~N} 8 \cdot 75$.

Das $\beta$-Äthylrhodanin ist unlöslich in Wasser und Petroläther, leicht löslich in Alkohol, Äther, Aceton und Eisessig 
schon in der Kälte; der Schmelzpunkt liegt bei $105^{\circ}$. Wird die Substanz kurze Zeit mit Lauge gekocht, dann angesäuert, mit einem Tropfen verdünnter Eisenchloridlösungund darauf mit Ammoniak versetzt, so entsteht eine dunkelbraunrote Färbung, die wohl der durch die Spaltung entstandenen $a$-Sulfhydrylbuttersäure zuzuschreiben ist, die also eine ähnliche Reaktion gibt wie die Thioglykolsäure.

$\beta$-Äthyliden-y-phenylrhodanin.<smiles>CC=C1OC(=O)N(c2ccccc2)SS1</smiles>

Dieser Körper, der zu weiteren Reduktionsversucher dienen soll, wird leicht aus Phenylrhodanin und Paraldehyd durch Erhitzen in Eisessig dargestellt. Beim Eingieß3en des Reaktionsproduktes in Wasser scheidet sich die neue Verbindung bald krystallinisch ab und kann aus Alkohol unter Verwendung von Tierkohle umkrystallisiert werden. Man erhält feine Krystallschuppen vom Schmelzpunkt $123^{\circ}$, die unter dem Mikroskop dünne Tafeln von lichtgelber Farbe erkennen lassen.

Analyse:

$0.1461 \mathrm{~g}$ Substanz: $0.3001 \mathrm{~g}_{\mathrm{CO}_{2}}, 0.0532 \mathrm{~g} \mathrm{H}_{2} \mathrm{O}$.

$\mathrm{C}_{11} \mathrm{H}_{9} \mathrm{ONS}_{2}$ Ber. C $56 \cdot 13, \mathrm{H} 3 \cdot 86$.

Gef. C $56 \cdot 01, \mathrm{H} 4 \cdot 07$.

Die Substanz ist in Wasser und Petroläther unlöslich, löst sich aber leicht in den gebräuchlichen organischen Lösungsmitteln schon in der Kälte.

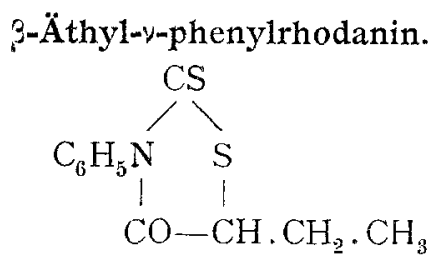

Dieser Körper, der durch Reduktion des zuletzt beschriebenen Rhodanins zu erwarten war, kann leicht durch 
Einwirkung von $\alpha$-Brombuttersäureäthylester auf phenyldithiocarbaminsaures Ammonium erhalten werden. Beide Verbindungen werden in äquimolekularen Mengen mit Alkohol einige Zeit im Sieden erhalten, das Reaktionsprodukt durch Wasser gefällt, das ausgeschiedene, übrigens bald krystallinisch erstarrende Öl mit Äther ausgeschüttelt und der Äther. rückstand zweimal aus Alkohol umkrystallisiert. Man erhält ein krystallinisches Pulver, das unter dem Mikroskop kurze Nadeln von schwach gelber Farbe aufweist; der Schmelzpunkt liegt bei $83^{\circ}$.

Analyse:

$0.1342 g$ Substanz: $0.2749 g \mathrm{CO}_{2}, 0.0576 g \mathrm{H}_{2} \mathrm{O}$.

$\mathrm{C}_{11} \mathrm{H}_{11} \mathrm{ONS}_{2}$ Ber. C $55 \cdot 66, \mathrm{H} 4 \cdot 6 \pi$. Gef. C $55 \cdot 68, \mathrm{H} 4 \cdot 70$.

Die Substanz ist in den gebräuchlichen organischen Lösungsmitteln leicht löslich, von Petroläther wird sie kaum aufgenommen.

Die Reduktionsversuche mit dem Äthylidenrhodanin und Äthylidenphenylrhodanin haben bisher noch keine befriedigenden Resultate ergeben. Aus letzterer Verbindung wurden durch Zinkstaub und Salzsäure in alkoholischer Lösung feine, schimmernde, weiße Blättchen vom Schmelzpunkt $205^{\circ}$ erhalten, aber in sehr geringer Menge; dieselben werden noch weiter untersucht werden.

\section{Phenyldithiocarbaminsäure-o-nitrobenzylester.}<smiles>O=[N+]([O-])c1ccccc1</smiles>

Dieser Körper wurde ebenfalls zu dem Zwecke von Reduktionsversuchen dargestellt. Man erhält ihn leicht in vorzüglicher Ausbeute durch Einwirkung ron o-Nitrobenzylchlorid auf phenyldithiocarbaminsaures Ammonium in alkoholischer Suspension. Nach kurzem Erwärmen am Rückflußkühler hat sich ein Krystallbrei von Salmiak ausgeschieden. Durch Zusatz von Wasser zur Reaktionsflüssigkeit geht der 
Salmiak in Lösung, während sich ein dickes gelbes Öl ausscheidet, das in der Kälte bald erstarrt. In der Flüssigkeit sind zahlreiche feine Krystallflitter $\mathrm{zu}$ bemerken. Der abgetrennte Krystallkuchen wird aus heißem Alkohol umkrystallisiert; dabei hat der Körper gerne die Neigung, sich zunächst als Öl abzuscheiden. Durch Einsäen der oben elwähnten Flitter kann es leicht zum Krystallisieren gebracht werden. In reinem Zustande stellt die Verbindung schwefelgelbe, aus feinen Nadeln bestehende Krystalldrusen dar, die bei 120 bis $121^{\circ}$ schmelzen. Zur Analyse wurde die Substanz bei $100^{\circ}$ getrocknet.

Analyse:

$0.2522 \mathrm{~g}$ Substanz: $0.510 \mathrm{~g} \mathrm{CO}_{2}, 0.0911 \mathrm{~g} \mathrm{H}_{2} \mathrm{O}$.

$0.1995 \mathrm{~g}$ Substanz $\left(24^{\circ}, 726 \mathrm{~mm}\right): 14.8 \mathrm{~cm}^{3}$.

$\mathrm{C}_{14} \mathrm{H}_{12} \mathrm{O}_{2} \mathrm{~N}_{2} \mathrm{~S}_{2}$ Ber. C $55 \cdot 23, \mathrm{H} 3 \cdot 98$. N $9 \cdot 21$.

Gef. C $55 \cdot 15, \mathrm{H} \pm \cdot 07, \mathrm{~N} 9 \cdot 35$.

Die Substanz ist in Alkohol und Äther und besonders in Aceton schon in der Kälte leicht löslich, etwas weniger leicht in Eisessig und Benzol, gar nicht in Petroläther. Mit der Substanz wurde auch eine Bestimmung des Molekulargewichtes nach der Methode der Siedepunktserhöhung ausgeführt.

Lösungsmittel Benzol, Siedepunkt $82^{\circ}$, Konstante $26 \cdot 7$.

Gewicht

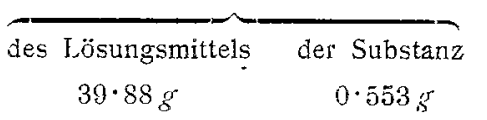

Erhöhung des Siedepunktes

$0 \cdot 11^{\circ}$
Molekulargewicht

Gefunden Bereclinet 324 301

\section{Methylendioxy-z-sulf hydrylzimtsäure.}

$$
\mathrm{CH}_{2} \cdot \mathrm{O}_{2} \cdot \mathrm{C}_{6} \mathrm{H}_{3} \cdot \mathrm{CH}: \mathrm{C}(\mathrm{SH}) \cdot \mathrm{COOH}
$$

Mit den verschiedensten Aldehydkondensationsprodukten der Rhodanine wurden Spaltungsversuche mit Alkalien (Barytwasser, Natronlauge, alkoholische Lauge) vorgenommen; während nun ein Teil dieser Verbindungen schon beim 
Erwärmen mit der Base im Wasserbad zerfällt, setzten andere Verbindungen, insbesondere solche mit einer Hydroxylgruppe im Phenylrest, der Spaltung größeren Widerstand entgegen. Durch die längere Einwirkung des Alkalis werden dann die Spaltungsprodukte meist selbst weiter abgebaut, so daß keine faßbaren Körper erhalten werden können. Bei einem Reduktionsversuch mit Natrium in Amylalkohol bin ich darauf gekommen, daß sich Natriumamylat für solche Spaltungsversuche gut eignet, wobei hauptsächlich die höhere Temperatur der Einwirkung $\left(132^{\circ}\right)$ maßgebend sein dürfte.

Es wurde zunächst das Kondensationsprodukt von Piperonal mit Phenylrhodanin ${ }^{1}$ zur Spaltung benutzt.

$2 g$ Natrium (= zwei Äquivalente) wurden in $25 \mathrm{~g}$ Amylalkohol gelöst und dazu die siedende Aufschlemmung ron $11 \mathrm{~g}$ Methylendioxbenzalphenylrhodanin in etwa der zehnfachen Menge Amylalkohol gegossen. Dabei tritt vollständige Lösung des Rhodanins ein; bei kurzem Kochen am Rückflußkühler beginnt sich ein weißgelber Niederschlag auszuscheiden, der deutlich krystallinisch ist und sich besonders an den geriebenen Glaswandstellen absetzt; nach 20 Minuten langem Kochen versetzt man langsam mit Wasser, wodurch zwei Schichten entstehen, beide von schmutziggelber Farbe. Die wässerige Schichte wird von dem aufschwimmenden Amylalkohol getrennt und die Flüssigkeit.zur Entfernung des Amylalkohols mit Äther ausgeschüttelt. Zusatz von Salzsäure erzeugt in der stark alkalischen Flüssigkeit einen weißgelben Niederschlag, der durch Ausschütteln mittels Äther in diesen übergeführt wird. Die Ätherauszüge von schwach gelber Farbe werden teilweise abdestilliert, worauf. beim Erkalten ein krystallinischer Niederschlag von fast chromgelber Farbe ausfällt, der nach dem Waschen mit etwas Äther analysiert wurde. Zur Analyse wurde die. Substanz bei $90^{\circ}$ getrocknet. Analyse:

$0.1419 g$ Substanz: $0.2785 \mathrm{~g} \mathrm{CO}_{2}, 0.048 \mathrm{~g}^{\prime} \mathrm{H}_{2} \mathrm{O}$.

$0.1021 \mathrm{~g}$ Substanz: $0.108 \mathrm{~g} \mathrm{BaSO}_{4}$.

$\mathrm{C}_{10} \mathrm{H}_{8} \mathrm{O}_{4} \mathrm{~S}$ Ber. C $53 \cdot 55, \mathrm{H} 3 \cdot 60$, S $14 \cdot 30$.

Gef. C $53 \cdot 52$. H $3 \cdot 64$, S $14 \cdot 40$.

1 R. Andreasch und A. Zi pser, Monatshefte füi Chemie, 24, 499. 
Die Substanz gibt, in Wasser verteilt, mit einem Tropfen verdünnten Eisenchlorids und etwas Ammoniak versetzt, eine dunkelgrüne Färbung, ein Beweis für das Vorhandensein der Sulfhydrylgruppe. Der Körper ist unlöslich in Wasser, in Alkohol besonders in der Wärme leicht löslich, desgleichen in Eisessig und Aceton, sehr wenig löslich in siedendem Benzol, unlöslich in Petroläther. Aus der heißen alkoholischen Lösung scheidet sich die Säure als feines gelbes Pulver ab, das unter dem Mikroskop sehr gleichmäßige, dünne, gelbe Nadeln erkennen läßt, die zu kugelartigen Aggregaten zusammengelegt sind. Beim Erhitzen im Kapillarrohr beginnt die Substanz sich von $170^{\circ}$ an zu zersetzen, wie aus dem Auftreten eines Sublimates erkennbar ist, ein vollständiges Schmelzen tritt erst bei 208 bis $210^{\circ}$ ein.

Zur Molekulargewichtsbestimmung wurde Eisessig verwendet.

Siedepunkt $117^{\circ}$, Konstante $25 \cdot 3$.

\begin{tabular}{|c|c|c|c|c|}
\hline \multicolumn{2}{|c|}{ Gewicht } & \multirow{2}{*}{$\begin{array}{l}\text { Exhöhung des } \\
\text { Siedepuniktes }\end{array}$} & \multicolumn{2}{|c|}{ Molekulargewicht } \\
\hline $\begin{array}{c}\text { ösungst } \\
30 g\end{array}$ & $\begin{array}{c}\text { der Substan } \\
0.45 g\end{array}$ & & $\begin{array}{l}\text { gefunden } \\
236\end{array}$ & $\begin{array}{c}\text { berechn } \\
224\end{array}$ \\
\hline
\end{tabular}

Der obenerwähnte, von der wässerigen Schichte abgetrennte Amylalkohol wurde fraktioniert. Nach Übergehen des enthaltenen Wassers destillierte der größte Teil beim Siedepunkt des Amylalkohols $\left(132^{\circ}\right)$ über, dann stieg das Thermometer rasch auf $220^{\circ}$, bei welcher Temperatur eine geringe Menge eines öligen Körpers überging, der den Geruch des Phenylsenföls zeigte. Mit alkoholischem Ammoniak zusammengebracht, trat Lösung ein und es konnte durch Verdampfen leicht Phenylthioharnstoff durch seine Eigenschaften und den Schmelzpunkt von $154^{\circ}$ erkannt werden.

Das Methylendioxybenzalphenylrhodanin zerfällt demgemäß unter dem Einfluß des Amyiates unter Wasseraufnahme nach der Gleichung:

$$
\begin{aligned}
& \mathrm{C}_{6} \mathrm{H}_{5} \mathrm{~N} . \mathrm{CS} . \mathrm{S} \\
& \mathrm{CO}-\mathrm{C}: \mathrm{CH} . \mathrm{C}_{6} \mathrm{H}_{3} \mathrm{O}_{2} \mathrm{CH}_{2} \\
& \quad=\mathrm{H}_{2} \mathrm{O}= \\
& \quad \mathrm{C}_{6} \mathrm{H}_{5} \mathrm{~N} \mathrm{C} \mathrm{S}+\mathrm{CH}_{2} \mathrm{O}_{2} \mathrm{C}_{6} \mathrm{H}_{3} \mathrm{CH}: \mathrm{C}(\mathrm{SH}) \mathrm{COOH}
\end{aligned}
$$

in Phenylsenföl und Methylendioxysufhyrdryizimtsäure. 
Die alkoholische Lösung der Säure entfärbt sofort alkoholische Jodlösung; wird von letzterer oder noch besser von einer Lösung von Jod in Jodwasserstoffsäure so lange zugesetzt, als noch Entfärbung eintritt, so trübt sich die Flüssigkeit alsbald unter Abscheidung eines gelben krystallinischen Niederschlages, der unter dem Mikroskop aus langen flachen Nadeln mit schief abgeschnittenen Enden besteht. Abfiltriert stellt die Substanz einen cadmiumgelben lockeren Krystallfilz dar. Bei der Umsetzung mit der Jodlösung wird genau ein Aquivalent Jod verbraucht, wie folgender Titrationsversuch ausweist.

$0.2232 \mathrm{~g}$ verbrauchten bis zur Bläuung eines mit Stärkekleister getränkten Papieres $43.5 \mathrm{~cm}^{3}$ einer Jodlösung, die in $1 \mathrm{~cm}^{3} 2.933 \mathrm{mg}$ Jod enthielt.

Verbraucht $127 \cdot 6 \mathrm{mg}$ Jod, berechnet $126 \cdot 5 \mathrm{mg}$.

Der Schmelzpunkt der neuen Substanz liegt bei $228^{\circ}$. Die Analyse bewies, daß die erwartete Methylendioxydisulfidzimtsäure vorlag:

$$
\begin{gathered}
\mathrm{CH}_{2} \mathrm{O}_{2} \mathrm{C}_{6} \mathrm{H}_{3} \mathrm{CH}=\mathrm{C}-\mathrm{COOH} \\
\mathrm{S} \\
\vdots \\
\mathrm{CH}_{2} \mathrm{O}_{2} \mathrm{C}_{6} \mathrm{H}_{3} \mathrm{CH}=\mathrm{C}-\mathrm{COOH}
\end{gathered}
$$

Analyse:

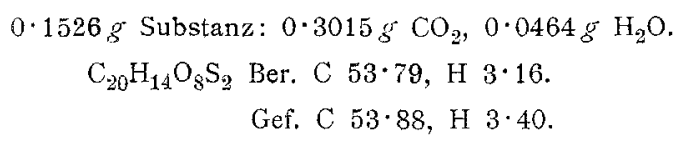

Die Säure löst sich in siedendem Alkohol mit schwach gelber Farbe, sehr wenig, auch in der Siedehitze, ist sie in Benzol und Eisessig, noch weniger in Aceton, fast gar nicht in Äther löslich. Am besten wird sie von Benzoesäureäthylester auch schon in der Kälte aufgenommen. 


\section{p-Oxy-m-methoxy- $\alpha$-sulf hydrylzimtsäure. $\mathrm{OH} . \mathrm{OCH}_{3} \mathrm{C}_{6} \mathrm{H}_{3}$. CH : C (SH) $\mathrm{COOH}$}

Diese Säure wurde durch Spaltung des aus Vanillin und Phenylrhodanin hergestellten Kondensationsproduktes ${ }^{1}$ mittels Natriumamylats hergestelit.

Es wurden einerseits $13 g$ der Verbindung mit Amylalkohol übergossen und zum Sieden erhitzt, ohne daß die Substanz vollkommen gelöst worden wäre, andrerseits wurde eine heiße Lösung von zwei Äquivalenten Natrium (2 statt der berechneten $1.9 \mathrm{~g}$ ) in Amylalkohol hergestellt und beide Lösungen vermischt. Unter dunkelblutroter Färbung der Flüssigkeit trat alsbald vollständige Lösung des Rhodanins ein; da selbst nach längerem Kochen die Farbe bestehen blieb, wahrscheinlich eine Folge der nichtsubstituierten Hydroxylgruppe, so wurde noch ein Äquivalent Natrium zugegeben. Jetzt verschwand die Färbung sofort und machte einer gelben Platz. Auf Zusatz von Wasser bildeten sich wieder zwei Schichten, die untere wässerige Schicht wurde nach Entfernung des gelösten Amylalkohols mittels Äthers mit Säure versetzt, wobei sich ein dicklicher bräunlicher Niederschlag ausschied. Nun wurde, ohne den Niederschlag abzutrennen, mit Äther ausgeschüttelt und der Äther zum Teil abdestilliert. Beim Erkalten schied sich ein schweres krystallinisches Pulver aus, das sich unter dem Mikroskop aus kurzen dicken Tafeln von rhombischem Umriß zusammengesetzt erwies. Die Substanz sintert beim Erwärmen im Kapillarrohr von $170^{\circ}$ an und ist bei $183^{\circ}$ vollkommen geschmolzen. Die Farbe der Säure ist lichtchromgelb.

Analyse:

$0 \cdot 1442 g$ Substanz: $0 \cdot 2804 g^{\circ} \mathrm{CO}_{2}, 0.0594 g \mathrm{H}_{2} \mathrm{O}$.

$\mathrm{C}_{10} \mathrm{H}_{10} \mathrm{O}_{4} \mathrm{~S}$ Ber. C $53 \cdot 08, \mathrm{H} 4 \cdot 46$.

Gef. C $53 \cdot 02$, H $4 \cdot 60$.

Die Lösungsverhältnisse der neuen Sulfhydrylzimtsäure sind dieselben wie die der vorhergehend beschriebenen

1 Andreasch und Zipser, Monatshefte für Chemie, 25, 163. 
Verbindung. Mit Eisenchlorid und Ammoniak gibt sie eine mehr schmutzig olivengrüne »Thioglykolsäurereaktion «. Setzt man Jodlösung zur alkoholischen Säurelösung, so wird diese sofort entfärbt. Die entstandene Disulfidsäure ist aber in Alkohol leicht löslich und konnte nicht in analysenreinem Zustande erhalten werden, weshalb auf ihre weitere Untersuchung verzichtet werden mußte.

\section{o-Oxy-x-sulf hydrylzimtsäure.$$
\left.\mathrm{OH} . \mathrm{C}_{6} \mathrm{H}_{4} \cdot \mathrm{CH}: \mathrm{C}(\mathrm{SH}) \mathrm{COOH}\right) \text {. }
$$

Zur Herstellung dieser Säure, beziehungsweise ihres Benzylderivates wurde das $o$-Oxybenzalphenylsenfölglykolid ${ }^{1}$ benutzt, das mit aikoholischer Kalilauge zersetzt wurde. Das Verfahren mit dem Natriumamylat hatte ich zur Zeit der Ausführung dieser Versuche noch nicht aufgefunden. Da sich außer der obigen Säure noch Anilin und Kohlendioxyd bilden mußten, so wurden etwas mehr als vier Äquivalente Ätzkali zur Zersetzung genommen. Wird die heiße Lauge in eine Aufschwemmung des Glykolids in Alkohol gegossen, so tritt sofort eine dunkelorangerote Färbung ein, wohl weil mit der freien Hydroxylgruppe zunächst ein Kalisalz des Glykolids entsteht. Beim Erwärmen am Rückflußkühler beginnt bald die Ausscheidung eines krystallinischen Niederschlages; sobald die Färbung mehr gelb geworden ist, wird die Flüssigkeit mit Wasser verdünnt und mit Salzsäure angesäuert. Unter starker Entwicklung von Kohlensäure scheidet sich eis bräunlich gefärbtes Ö1 ab, das durch Ausschütteln mit Äther in diesen übergeführt wird. Nach Abdestillieren des Äthers hinterbleibt ein dickliches, fast terpentinartiges Öl, das keine Neigung zum Krystallisieren zeigte. Das Ö1 hat einen schwachen eigentümlichen Geruch, der gleichzeitig an Zimtöl und gewisse Schwefelverbindungen erinnert. Auffallend war, daß das aus der Lauge abgeschiedene Öl sich jetzt in Lauge nur mehr langsam löste; erst bei längerem Stehen oder Elwärmen trat vollständige Lösung ein. Es macht dies

1 Andreasch, Monatshefte für Chemie, $38,127$. 
den Eindruck, daß bereits in diesem Öl mindestens teilweise ein inneres Anhydrid, also ein Cumarin, vorlag, dem wohl die Formel<smiles></smiles>

zukommt. Mit Eisenchlorid und Ammoniak versetzt, zeigte das Ö1 eine prächtige smaragdgrüne Färbung, ein Beweis für die intakte Sulfhydrylgruppe. Da eine Analyse des Öles als solches aussichtslos war, wurde die Sulfhydrylzimtsäure in ihr Benzylderivat übergeführt. (Nach Suter, Zeitschrift für physiol. Chemie, 20, 562). Aus der alkalischen Lösung wurde die Benzylverbindung als anfangs teigige, bald krystallinisch werdende Masse gefällt. Durch Umkrystallisieren aus Alkohol unter Verwendung von Tierkohle wurden feine schmutzigweiße Nädelchen erhalten, die zu lockeren Drusen zusammengesetzt waren. Da die Analyse anfangs nicht zu der erwarteten Zusammensetzung einer Oxybenzylsulfhydrylzimtsäure stimmte, wurde die Substanz nochmals umkrystallisiert, doch änderte sich die Zusammensetzung dabei nicht, Es zeigte sich vielmehr, daß nicht mehr die vermutete Säure, sondern bereits ihr Anhydrid, das entsprechende Cumarin<smiles>CCC(C)(CC)C(=O)OCc1ccccc1</smiles>

das man wohl als Benzyl- $\beta$-sulfhydrylcumarin bezeichnen kann, vorlag. Die zweite der unten angeführten Analysen ist übrigens mit einem Präparat ausgeführt worden, das durch Barytspaltung usw. aus dem 0 -Oxybenzalphenylsenfölglykolid erhalten worden war. Der Schmelzpunkt der Verbindung liegt bei 164 bis $165^{\circ}$.

Analyse:

$0.1197_{g}$ Substanz: $0.3141 \mathrm{~g} \quad \mathrm{CO}_{2}, \quad 0.0497 \xi \mathrm{H}_{2} \mathrm{O} .0 .1401 g$ Substanz: $0.367 \mathrm{~g} \quad \mathrm{CO}_{2}, \quad 0.0564 \mathrm{~g} \quad \mathrm{H}_{2} \mathrm{O} .0 .1651 \%$ Substanz: $0.4311 \mathrm{~g} \quad \mathrm{CO}_{2}$, $0.0695 \mathrm{~g} \mathrm{H}_{2} \mathrm{O}$. $0.124 \mathrm{~g}$ Substanz: $0.1085 \mathrm{~g} \mathrm{BaSO}_{4}$. $\mathrm{C}_{16} \mathrm{H}_{12} \mathrm{O}_{2} \mathrm{~S}$ Ber. C $71 \cdot 61, \mathrm{H} 4 \cdot 51$, S $11 \cdot 95$.

Gef. C $71 \cdot 56,71 \cdot 43,71 \cdot 20 ; \mathrm{H} 4 \cdot 64,4 \cdot 50,4 \cdot 71 ; \mathrm{S} 12 \cdot 01$. 
Das Benzylsulfhydrylcumarin ist in Wasser unlöslich, in Alkohol ist es besonders in der Siedehitze etwas löslich, ebenso in Eisessig, beim Erkalten auskrystallisierend; auch von Aceton wird der Körper leicht aufgenommen, dagegen ist er in Benzol auch beim Kochen nur wenig, in Petroläther gar nicht löslich. Die alkoholische Lösung reagiert neutral, Helianthin wird dadurch nicht verändert, eine durch Alkali rot gefärbte Phenolphthaleinlösung nicht entfärbt. In Lauge ist die Substanz nicht mehr löslich, wird aber sehr starke (50prozentige) Lauge verwendet, so tritt nach einiger Zeit Lösung ein, Säure fällt wieder unverändertes Cumarin.

\section{Furylsulfhydrylakrylsäure.}

$$
\mathrm{C}_{4} \mathrm{H}_{3} \mathrm{O} . \mathrm{CH}: \mathrm{C}(\mathrm{SH}) . \mathrm{COOH}
$$

Zur Spaltung diente das Furfuralphenylrhodanin. ${ }^{1}$ Dabei wurde der Versuch gemacht, das unbequeme Arbeiten mit dem Amylalkohol zu umgehen, indem derselbe durch Äthylalkohol ersetzt wurde. Beim Vermischen der Flüssigkeiten und kurzem Kochen setzt sich ein lehmfarbener Niederschlag $a b$, der unter dem Mikroskop feine garbenförmige Nadelbüschel ausweist. Nach Abdestillieren eines Teiles des Alkohols wurde die Lösung mit dem Niederschlag mit Säure versetzt, der ausgefallene Niederschlag in Äther aufgenommen und das Lösungsmittel abdestilliert. Durch Aufstreichen auf Tonteller konnten schmierige Nebenprodukte entfernt werden, worauf die zurückbleibenden Krystalle aus Alkohol unter Zuhilfenahme von Tierkohle umkrystallisiert wurden. Es resultierten prächtige weiße Krystallnadeln vom Schmelzpunkt $71^{\circ}$. Bei der näheren Untersuchung zeigte sich aber, daß nicht die gesuchte Furylsulfhydrylakrylsäure vorliegen konnte, denn der erhaltene Körper erwies sich als stickstoffhaltig. Die Analyse ergab nun Zahlen, die zur Formel des. Phenylthiocarbamats des Äthyls (Phenylthiourethan, Xanthogenanilid) führten. 
Analyse:

$0.1383 g$ Substanz: $0.3023 \% \quad \mathrm{CO}_{2}, \quad 0.0759 \% \mathrm{H}_{2} \mathrm{O}, 0.1525 \xi$ Substanz: $0.3325 \mathrm{~g} \mathrm{CO}_{2}, 0.0846 \mathrm{~g} \mathrm{H}_{2} \mathrm{O} .0 .136 \mathrm{~g}$ Substanz: $0.1759 g \mathrm{BaSO}_{1}$. $\mathrm{C}_{2} \mathrm{H}_{11}$ ONS Ber. C $59 \cdot 63$, H 6.12, S $17 \cdot 69$.

Gef. C $59 \cdot 61,59 \cdot 59, \mathrm{H} 6 \cdot 14,6 \cdot 27$, S $17 \cdot 76$.

Dieser Körper, dem die Konstitution $\mathrm{C}_{6} \mathrm{H}_{5} \mathrm{NH}$. CS.O. $\mathrm{C}_{2} \mathrm{H}_{5}$ zukommt, ist bereits von $\mathrm{A}$. W. Hofmann aus Phenylsenföl und Alkohol dargestellt worden, während Schiff nachwies. daß der Körper leicht bei der Einwirkung von alkoholischem Kali auf Phenylsenföl entsteht. ${ }^{1}$ Auch in unserem Falle ist der Körper durch die Einwirkung der alkoholischen Kalilauge auf das durch Spaltung entstandene Phenylsenföl gebildet worden, während das zweite Spaltungsprodukt, die Furfuralthioglykolsäure, dem Nachweis entgangen war.

Da nun die Spaltung des Furfuralphenylrhodanins mit Natriumäthylat nicht gelungen war, wurde das Furfuralrhodanin ${ }^{2}$ der Barytspaltung unterworfen und dabei wesentlich nach der Vorschrift verfahren, die von Bondzyńki für die Spaltung des Benzalrhodanins angewandt worden war. ${ }^{3}$

Die feingeriebene Substanz wurde mit der fünffachen Menge Barythydrat und dem 20 fachen Gewicht an Wasser im siedenden Wasserbad erwärmt. Als sich alles bis auf einen kleinen Rest von kohlensaurem Baryt gelöst hatte (etwa nach dreiviertel Stunden), wurde die filtrierte Lösung mit Salzsäure ausgefällt und der gelblichbräunliche Niederschlag in Äther aufgenommen. Die vereinigten Ätherauszüge wurden abdestilliert und der Rückstand aus Wasser unter Anwendung von Tierkohle umkrystallisiert. Das Filtrat trübt sich anfangs milchig, später verwandelt sich die Trübung in Krystalle, die, abfiltriert, feine Nadeln von schmutziggelber Farbe darstellen. Der Schmelzpunkt liegt bei 102 bis $103^{\circ}$. Analyse:

$0 \cdot 1297 g$ Substanz: $0 \cdot 2348 g \mathrm{CO}_{2}, 0 \cdot 0402 \& \mathrm{H}_{2} \mathrm{O}$.

$\mathrm{C}_{7} \mathrm{H}_{6} \mathrm{O}_{3} \mathrm{~S}$ Ber. C $49 \cdot 39, \mathrm{H} 3 \cdot 55$.

Gef. C $49 \cdot 29$, H $3 \cdot 46$.

1 Ber. d. d. chem. Ges., 9, 1316.

2 Andreasch und Zipser, Monatshefte für Chemie, 26, 1201.

3 Ibid., 8,350 . 
Die Furfylsulfhydrylakrylsäure ist außer in Wasser auch in Alkohol, Aceton, Eisessig und Benzol schon in der Kälte ziemlich leicht löslich, am wenigsten noch in letzterem Lösungsmittel. Petroläther löst nicht, die Benzollösung wird durch Zusatz von Petroläther krystallinisch gefällt. Wird eine kleine Menge der Säure in Wasser gelöst und mit Eisenchlorid und Ammoniak versetzt, so tritt eine blaue Färbung ein, die einer kolloidalen Goldlösung ähnelt. Es gibt also auch diese Säure eine »Thioglykolsäurereaktion «.

Wird die Säure in heißem Alkohol gelöst und dazu eine Lösung von Jod in Jodwasserstoffsäure gegeben, so verschwindet das Jod sofort und nach einiger Zeit beginnen sich Krystalle auszuscheiden, die unter dem Mikroskop flache langgestreckte Nadeln oder Tafeln von sechsseitigem Umriß darstellen und häufig zu lockeren Drusen zusammengelagert sind. Auch aus der alkoholischen Lösung läßt sich ein weiterer Anteil durch Abdestillieren des Lösungsmittels gewinnen. Die Substanz läßt sich aus Alkohol umkrystallisieren, sie ist auch in den anderen organischen Lösungsmitteln löslich, aber entschieden schwerer als ihre Muttersubstanz. Die Verbindung schmilzt bei 190 bis $191^{\circ}$. Die Farbe ist ein reines Zitronengelb. Die Analyse erwies, daß die vermutete Disulfidverbindung

$$
\begin{aligned}
& \mathrm{C}_{4} \mathrm{H}_{3} \mathrm{O} . \mathrm{CH}: \mathrm{C} . \mathrm{COOH} \mathrm{S}_{2} \\
& \mathrm{C}_{4} \mathrm{H}_{3} \mathrm{O} . \mathrm{CH}: \mathrm{C} . \mathrm{COOH}
\end{aligned}
$$

vorlag. Die Namengebung stößt hier auf Schwierigkeiten, vielleicht könnte man den Körper am besten Difuryl- $\alpha-\mathrm{di}$ sulfidakrylsäure nennen.

Analyse:

$0 \cdot 1223 \mathrm{~g}$ Substanz: $0 \cdot 2231 \mathrm{~g} \mathrm{CO}_{2}, 0.0341 \mathrm{~g} \mathrm{H}_{2} \mathrm{O}$.

$\mathrm{C}_{14} \mathrm{H}_{10} \mathrm{O}_{6} \mathrm{~S}_{2}$ Ber. C $49 \cdot 68$, H 2.98.

Gef. C $49 \cdot 75, \mathrm{H} 3 \cdot 12$.

Die Molekulargewichtsbestimmung beider Säuren wurde nach der Methode der Siedepunktserhöhung im Beckmann'schen Apparat vorgenommen. 
Lösungsmittel Aceton, Siedepunkt $56^{\circ}$, Konstante $17 \cdot 1$.

\begin{tabular}{|c|c|c|c|c|}
\hline \multicolumn{2}{|c|}{ Gewicht } & \multirow{2}{*}{$\begin{array}{l}\text { Erhöhung des } \\
\text { Siedepunktes }\end{array}$} & \multicolumn{2}{|c|}{ Molekulargewicht } \\
\hline es Lösungsmittels & der Substanz & & gefunden & berechnet \\
\hline $30 g$ & $0 \cdot 4069 \mathrm{~g}$ & $0 \cdot 13^{\circ}$ & 177 & 170 \\
\hline $30 \mathrm{~g}$ & $0.340 \mathrm{~g}$ & $0 \cdot 06^{\circ}$ & 323 & 338 \\
\hline
\end{tabular}

Die Molekulargewichtsbestimmung ergibt also einen mit der angenommenen Konstitution übereinstimmenden Wert; eine Eisenreaktion gibt die Säure, wie vorauszusehen, nicht. Ein weiterer Spaltungsversuch wurde mit dem $p$-Oxybenzalrhodanin ausgeführt. Diese Verbindung ist bereits von Bargellini1 aus Rhodanin und p-Oxybenzaldehyd durch Kondensation mittels Schwefelsäure in alkoholischer Lösung hergestellt worden. Ich benutzte die Methode des Erhitzens der Komponenten in Eisessiglösung. Das Oxybenzalrhodanin krystallisiert aus Alkohol in gelben oder bräunlichen Nadeln mit schwach blauer Oberflächenfarbe. Es ist in kochendem Wasser in Spuren löslich, leichter wird es von Alkohol in der Siedehitze aufgenommen, durch Wasserzusatz. fällt es wieder als cadmiumgelber Niederschlag aus. Der Körper ist auch in Äther und Eisessig nur wenig, etwas leichter in Aceton löslich, gar nicht wird er von Benzol und Petroläther aufgenommen. Die Substanz sintert von $260^{\circ}$ an, ist aber erst bei $274^{\circ}$ vollständig geschmolzen.

Analyse:

4.045 mg Substanz: $7 \cdot 463 m_{\mathrm{g}} \mathrm{CO}_{2}, 1 \cdot 140 \mathrm{mg}_{\mathrm{g}} \mathrm{H}_{2} \mathrm{O} .1$

$\mathrm{C}_{10} \mathrm{H}_{7} \mathrm{O}_{2} \mathrm{NS}_{2}$ Ber. C 50.60, $\mathrm{H} 2_{2} \cdot 97$.

Gef. C $50 \cdot 32, \mathrm{H} 3 \cdot 15$.

Spaltungsversuch mit Barythydrat. $20 \mathrm{~g}$ 'der Verbindung wurden mit $80 \mathrm{~g}$ Barythydrat und $500 \mathrm{~cm}^{3}$ Wasser im kochenden Wasserbad erwärmt. Anfangs fällt ein krystallini-

1 Atti R. Accad. dei Lincei Roma (5), 15, I, 35-43; Chem. Zentralbl., 1916, I, 1437.

2 Für die Ausführung dieser und der folgenden Mikroanalysen nach Pregl bin ich meinem Assistenten Herrn Dr. K. Stieger zu Dank verplichtet. 
scher Niederschlag heraus, offenbar das Barytsalz der unzersetzten Verbindung, gleichzeitig färbt sich die Flüssigkeit dunkelblutrot. Bei längerem Erhitzen geht der Niederschlag wieder in Lösung bis auf einen kleinen Rest, der sich als Bariumcarbonat erwies. Die Flüssigkeit wird nun mit Säure ausgefällt und der Niederschlag in Äther aufgenommen. Nach Abdestillieren des letzteren hinterblieb ein Öl, das erst nach mehreren Tagen eine kleine Menge warzenförmiger Krystalle absetzte, die sich aber nicht rein erhalten ließen. Die Hauptmenge des Öles wurde deshalb in zehnprozentiger Natronlauge gelöst und in bekannter Weise in die Benzylverbindung übergeführt. Nach Reinigung derselben über das Natronsalz und dem Umkrystallisieren aus etwa 50 prozentigem Alkohol wurden weiße mikroskopische Nadeln erhalten, die ein schimmerndes Krystallpulver darstellten. Die Substanz schmolz bei $183^{\circ}$.

Analyse:

$0.1376 g$ Substanz: $0.3392 g \mathrm{CO}_{2}, 0.0627 g \mathrm{H}_{2} \mathrm{O}$.

$0 \cdot 1455 \mathrm{~g}$ Substanz: $0 \cdot 1198 \mathrm{~g} \mathrm{BaSO}_{4}$.

$$
\begin{array}{r}
\mathrm{C}_{16} \mathrm{H}_{14} \mathrm{O}_{3} \mathrm{~S} \text { Ber. C } 67 \cdot 10 \text {, H } 4 \cdot 93 \text {, S } 11 \cdot 20 . \\
\text { Gef. C } 67 \cdot 22 \text {, H } 5 \cdot 10 \text {, S } 11 \cdot 30 .
\end{array}
$$

Es liegt also in der Tat die erwartete $p$-Oxybenzylsulfhydrylzimtsäure $\mathrm{OH} . \mathrm{C}_{6} \mathrm{H}_{4} \mathrm{CH}: \mathrm{C}\left(\mathrm{SC}_{7} \mathrm{H}_{7}\right) \mathrm{COOH}$ vor.

$\mathrm{Da}$ die Abscheidung der $p$-Oxysulfhydrylzimtsäure in diesem Versuch nicht gelungen war, wurde die Spaltung nochmals mit Natriumamylat wiederholt, dazu aber das bisher unbekannte

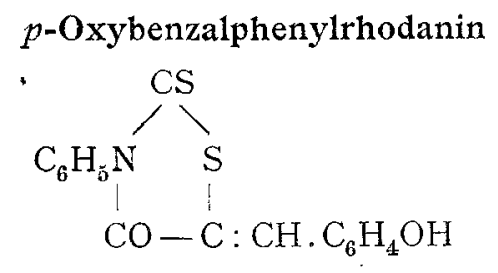

benutzt. Der Körper wird leicht durch Erhitzen der Komponenten Phenylrhodanin und $p$-Oxybenzaldehyd in Eisessig erhalten. Nach halbstündigem Sieden beginnt sich die Verbindung abzuscheiden, wodurch starkes Stoßen veranlaßt 
wird. Am besten unterbricht man jetzt den Prozeß, saugt nach dem Erkalten den Krystallbrei ab und erhitzt das Filtrat von neuem. Nach dem Umkrystallisieren aus Alkohol erhält man cadmiumgelbe Nadeln, die zu lockeren Büscheln vereinigt sind. Auch hier bemerkt man wieder die schwach blaue Oberflächenfarbe. Der Schmelzpunkt liegt bei $285^{\circ}$.

Analyse:

$0.680 \mathrm{mg}$ Substanz: $10.526 \mathrm{mg} \mathrm{CO}_{2,}, 1.509 \mathrm{mg} \mathrm{H}_{2} \mathrm{O}$.

$\mathrm{C}_{16} \mathrm{H}_{11} \mathrm{O}_{2} \mathrm{NS}_{2}$ Ber. C $61 \cdot 31, \mathrm{H} 3 \cdot 54$.

Gef. C $61 \cdot 37, \mathrm{H} 3 \cdot 60$.

Der Körper ist unlöslich in Wasser, ziemlich leicht löslich in Alkohol, besonders beim Sieden, in Eisessig und Äther ist die Substanz auch in der Hitze nur mäßig löslich mit schwach gelber Farbe, noch schwerer löslich ist sie in Chloroform, Benzol, gar nicht in niedrigsiedendem Petroläther.

Beim Vermischen der heißen amylalkoholischen Lösung mit Natriumamylat (zwei Atomen $\mathrm{Na}$ entsprechend) fällt sofort ein gelber Niederschlag aus; nach kurzem Erwärmen ist die Spaltung beendet. Die Aufarbeitung erfolgt wie in den früheren Fällen. Durch Umkrystallisieren des Rohproduktes aus verdünntem Alkohol werden aus feinen Nadeln zusammengesetzte Warzen von chromgelber Farbe erhalten, die einen Schmelzpunkt von $186^{\circ}$ aufweisen. Die Analyse zeigte, daß die gesuchte $p$-Oxysulfhydrylzimtsäure:

vorlag.

$$
\mathrm{OH} \cdot \mathrm{C}_{6} \mathrm{H}_{4} \cdot \mathrm{CH}: \mathrm{C}(\mathrm{SH}) \cdot \mathrm{COOH}
$$

Analyse:

$0.137 \mathrm{~g}$ Substanz: $0.2761 \mathrm{~g} \mathrm{CO}_{2}, 0.052 \mathrm{~g} \mathrm{H}_{2} \mathrm{O}$.

$\mathrm{C}_{9} \mathrm{H}_{8} \mathrm{O}_{3} \mathrm{~S}$ Ber. C $55 \cdot 08, \mathrm{H}_{4} \cdot 11$.

Gef. C $54 \cdot 96, \mathrm{H} 4 \cdot 25$.

Die Säure ist sehr leicht löslich in Alkohol, Aceton, Eisessig und Äther, unlöslich außer in Wasser in siedendem Chloroform, Benzol und Petroläther. Sie gibt eine dunkelgrüne $»$ Thioglykolsäurereaktion «.

Durch Versetzen der alkoholischen Lösung der Säure mit Jodlösung, Fällen mit Wassèr und Umkrystallisieren des 
Niederschlages aus Alkohol erhält man ein gelbes krystallinisches Pulver vom Schmelzpunkt $197^{\circ}$, das die betreffende Disulfidsäure $\left[\mathrm{HO} . \mathrm{C}_{6} \mathrm{H}_{4} \mathrm{CH}: \mathrm{C}(\mathrm{COOH}) \mathrm{S}\right]_{2}$, die man als $p-\mathrm{Oxy}-$ disulfidzimtsäure bezeichnen kann, darstellt.

Analyse:

4. $396 \mathrm{mg}$ Substanz: $9.353 \mathrm{mgr} \mathrm{CO}_{2}, 1.518 \mathrm{mg} \mathrm{H}_{2} \mathrm{O}$.

$\mathrm{C}_{18} \mathrm{H}_{14} \mathrm{O}_{6} \mathrm{~S}_{2}$ Ber. C $55 \cdot 35, \mathrm{H} 3 \cdot 62$.

Gef. C $55 \cdot 50$, H $3 \cdot 61$.

Ein anderer Teil der $p$-Oxysulfhydrylzimtsäure wurde nach bekannter Methode in die Benzylverbindung übergeführt und diese in allen Stücken mit der nach der Barytspaltung des Oxybenzalrhodanins erhaltenen identisch gefunden.

Analyse:

$0.1268 g$ Substanz: $0.311 g \mathrm{CO}_{2}, 0.0573 \xi \mathrm{H}_{2} \mathrm{O}$.

$\mathrm{C}_{16} \mathrm{H}_{14} \mathrm{O}_{3} \mathrm{~S}$ Ber. C $67 \cdot 10$, $\mathrm{H} 4 \cdot 93$.

Gef. C $67 \cdot 10, \mathrm{H} 5 \cdot 06$.

p-Dimethylaminosulf hydrylzimtsäure.

$\left(\mathrm{CH}_{3}\right)_{2} \mathrm{~N} \cdot \mathrm{C}_{6} \mathrm{H}_{4} \cdot \mathrm{CH}: \mathrm{C}(\mathrm{SH}) \cdot \mathrm{COOH}$

Zur Darstellung dieser Säure wurde das Dimethylaminobenzalphenylrhodanin ${ }^{1}$ der Hydrolyse durch Natriumamylat unterworfen. Beim Zusammenbringen der Lösungen fiel alsbald ein aus garbenförmigen Krystallbüscheln bestehender Niederschlag aus, der auf Zusatz von Wasser in Lösung ging. Die orangegefärbte alkalische Flüssigkeit gibt auf Zusatz von Säure einen hochgelben Niederschlag, der beim Umkrystallisieren aus verdünntem Alkohol die Farbe des gefällten Schwefelantimons annimmt. Der Schmelzpunkt liegt bei $160^{\circ}$. Analyse:

$0 \cdot 144 \mathrm{~g}$ Substanz: $0.3116 g \mathrm{CO}_{2}, 0.0759 \mathrm{~g} \mathrm{H}_{2} \mathrm{O}$.

$\mathrm{C}_{11} \mathrm{H}_{13} \mathrm{O}_{2} \mathrm{NS}$ Ber. C $59 \cdot 16, \mathrm{H} 5 \cdot 87$.

Gef. C $59 \cdot 01$, H $5 \cdot 90$.

Die Säure ist leicht löslich in Aceton, Eisessig und Äther und gibt eine schmutzigolivengrüne »Thioglykolsäurereaktion «.

1 Andreasch und Zipser, Monatshefte für Chemie, 26, 1205. 
Zusatz von Jodlösung zur alkoholischen Lösung der Säure bis zur Bläuung von Stärkepapier und Stehenlassen der Flüssigkeit oder noch besser Reiben mit einem Glasstab bewirkt die Ausscheidung der entsprechenden Disulfidsäure, die sich als scharlachrotes Pulver abscheidet. Der Schmelzpunkt liegt bei $198^{\circ}$.

Analyse:

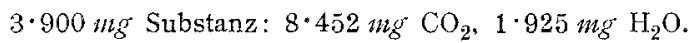

$\mathrm{C}_{22} \mathrm{H}_{24} \mathrm{O}_{4} \mathrm{~N}_{2} \mathrm{~S}_{2}$ Ber, C $59 \cdot 42, \mathrm{H} 5 \cdot 44$.

Gef. C $59 \cdot 11$, H $5 \cdot 52.1$

Die Säure zeigt unter dem Mikroskop. sehr schöne orangerote Tafeln, die häufig zu Durchdringungszwillingen vereinigt sind. Der Körper ist sehr wenig löslich in Aceton, Eisessig und Chloroform, am leichtesten löst er sich noch in kochendem Alkohol zur chromgelb gefärbten Flüssigkeit.

Für Spaltungsversuche wurden noch die beiden folgenden Verbindungen hergestellt:

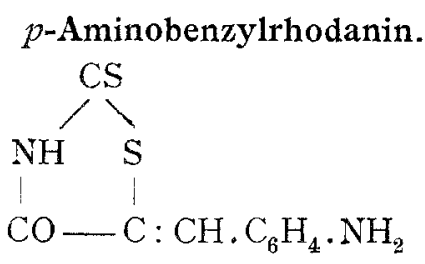

Der Körper entsteht leicht durch Erhitzen von Rhodanin mit $p$-Aminobenzaldehyd in Eisessiglösung. Schon bei kurzem Erhitzen färbt sich die Flüssigkeit dunkelrot und scheidet das Kondensationsprodukt als krystallinisches Pulver von dunkelbraunroter Farbe ab. Durch Umkrystallisieren aus Alkohol werden feine wollige Nadeln erbalten, die lebhaft an Chromtrioxyd erinnern. Die Substanz beginnt bei etwa $200^{\circ} \mathrm{zu}$ sintern und ist selbst bei $290^{\circ}$ noch nicht vollkommen geschmolzen.

Analyse:

$0 \cdot 1251 g$ Substanz: $0.2325 \mathrm{~g} \mathrm{CO}_{2}, 0.0403 \mathrm{~g} \mathrm{H}_{2} \mathrm{O}$.

$\mathrm{C}_{10} \mathrm{H}_{8} \mathrm{ON}_{2} \mathrm{~S}_{2}$ Ber. C $50 \cdot 81, \mathrm{H} 3 \cdot 41$.

Gef. C $50 \cdot 68, \mathrm{H} 3 \cdot 65$.

1 Die Substanz hinterließ im Schiffchen eine kleine Menge Asche. 
Die Substanz ist in Wasser und Petroläther unlöslich, löst sich ziemlich leicht in heißem Alkohol und Aceton, weniger in Eisessig, sehr wenig in Benzol. Das Aminobenzalrhodanin hat den Charakter eines Farbstoffes und färbt tierische Faserstoffe gelb.

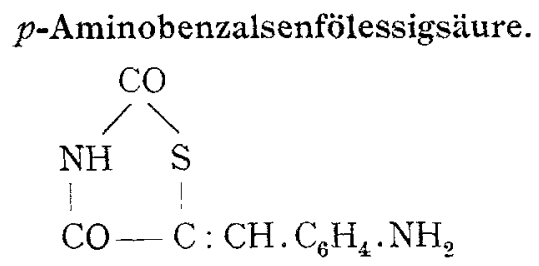

Der Körper wird auf analoge Art aus Senfölessigsäure und Aminobenzaldehyd erhalten. Er stellt (aus Alkohol) ein schmutzigrotbraunes Pulver dar, das keinen eigentlichen Schmelzpunkt aufweist, indem es sich von $200^{\circ}$ an immer dunkler färbt.

Analyse:

$0.1228 g$ Substanz: $0.245 g \mathrm{CO}_{2}, 0.0417 \mathrm{~g} \mathrm{H}_{2} \mathrm{O}$.

$\mathrm{C}_{10} \mathrm{H}_{8} \mathrm{O}_{2} \mathrm{~N}_{2} \mathrm{~S}$ Ber. C $54 \cdot 52, \mathrm{H} 3 \cdot 66$.

Gef. C $54 \cdot 41, \mathrm{H} 3 \cdot 8$.

Die Substanz ist auch in Eisessig und Aceton, wenn auch schwer, löslich.

Spaltungsversuche mit den beiden zuletzt beschriebenen Verbindungen führten zu keinen faßbaren Produkten, ebenso ergebnislos waren Versuche, das $o$-Nitrobenzalphenylrhodanin durch Natriumamylat zu spalten. Ich bin damit zu denselben negativen Ergebnissen gelangt, wie schon vor fast 30 Jahren St. Bondzyński, ${ }^{1}$ obwohl ich mit größeren Materialmengen (bis zu $20 \mathrm{~g}$ ) arbeitete.

Die Untersuchungen werden fortgesetzt.

1 Monatshefte für Chemie, $s, 349$. 